\title{
Two-year Optical Coherence Tomography Findings after Balloon-Only Treatment of Bioresorbable Scaffold Restenosis in a Calcified Coronary Lesion: A Case Report
}

\author{
Abdelhakim Allali $\cdot$ Mohamed Abdel-Wahab $\cdot$ Gert Richardt
}

Received: May 31, 2016 / Published online: July 29, 2016

(C) The Author(s) 2016. This article is published with open access at Springerlink.com

\begin{abstract}
We report a case of a 65 -year-old female patient treated with an everolimus-eluting bioresorbable scaffold (BRS) after rotational atherectomy of a severely calcified lesion of the left anterior descending coronary artery. The implanted BRS restenosed 10 months after implantation and was treated with a scoring balloon followed by drug-eluting balloons with good immediate and long-term results. To the best of our knowledge, this is the first report of a balloon-only treatment of a restenosis occurring more than 6 months after BRS implantation; angiographic and optical coherence tomography images after treatment and at two-year follow-up are presented.
\end{abstract}

Keywords: Bioresorbable vascular scaffold; Calcified coronary lesions; Drug eluting

Enhanced content To view enhanced content for this article go to http://www.medengine.com/Redeem/ 97E4F0601BA643AA.

A. Allali $(\bowtie) \cdot$ M. Abdel-Wahab · G. Richardt Heart Center, Segeberger Kliniken $\mathrm{GmbH}$, Academic Teaching Hospital for the Universities of Kiel, Lübeck and Hamburg, Bad Segeberg, Germany e-mail: abdelhakim.allali@segebergerkliniken.de balloon; Rotational atherectomy; Scoring balloon

\section{CASE REPORT}

A 65-year-old female patient presented in January 2013 with unstable angina. Coronary angiography showed a severely calcified long lesion of the proximal and mid segments of the left anterior descending coronary artery [Video S1 (please see enhanced content link on title page)]. In view of the angiographic findings, a decision was taken to do a planned rotational atherectomy (RA), which was performed with the Rotablator ${ }^{\mathrm{TM}}$ (Boston Scientific) using 1.5 then $1.75 \mathrm{~mm}$ burr sizes, followed by implantation of two overlapping $3.0 \times 28 \mathrm{~mm}$ bioresorbable scaffolds (BRS) (Absorb ${ }^{\mathrm{TM}}$, Abbott). Post-dilatation was done using a $3.0 \mathrm{~mm}$ non-compliant balloon at $20 \mathrm{~atm}$. Intravascular ultrasound (IVUS) showed a good result with a minimal overlapping zone (Fig. 1). A coronary angiography done 10 months later due to recurrent angina revealed a significant restenosis of the implanted BRS. Optical coherence tomography (OCT) showed a diffuse homogeneous intra-scaffold neointimal tissue with a minimum luminal area of $1.59 \mathrm{~mm}^{2}$ at 

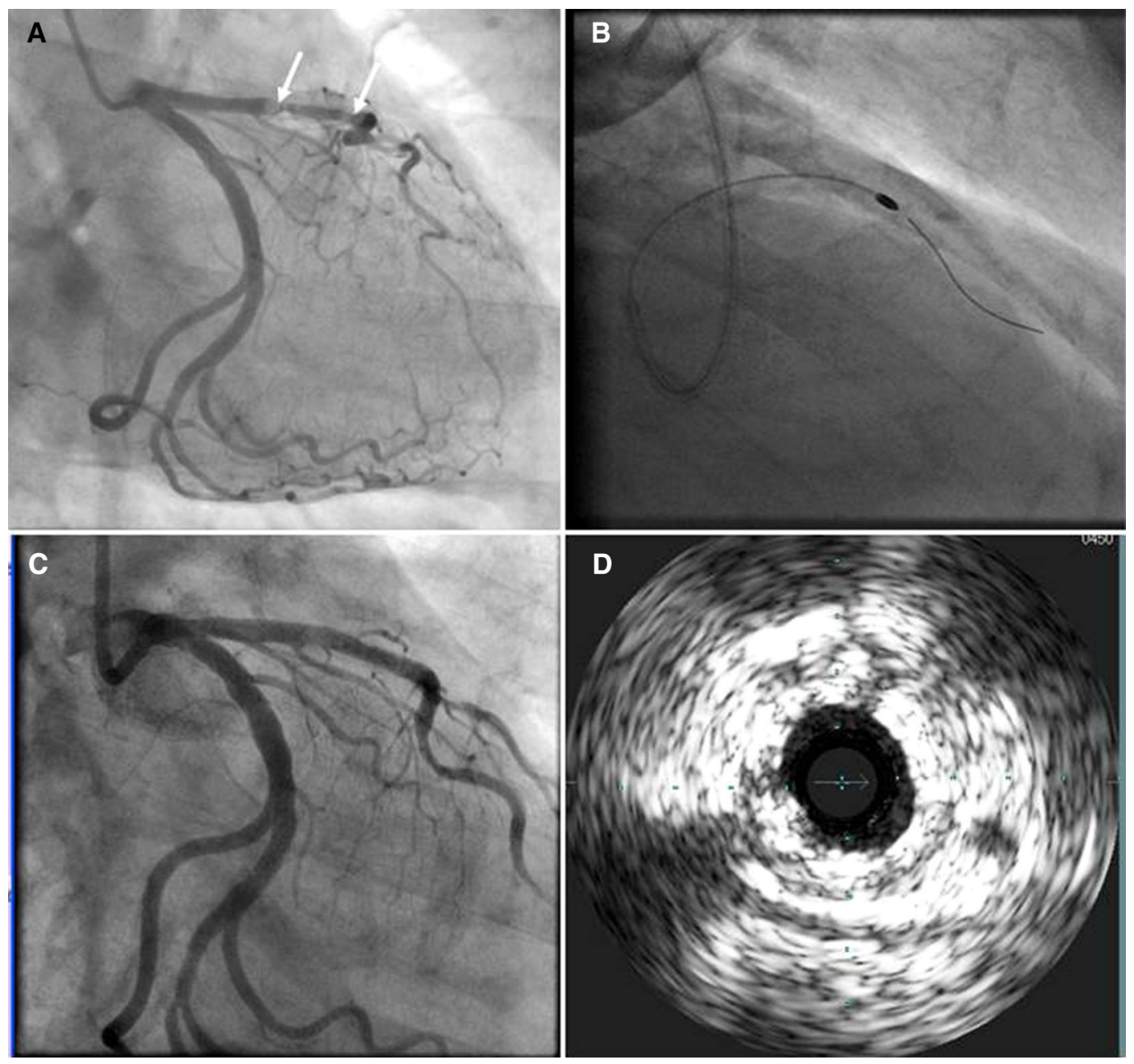

Fig. 1 Baseline angiogram and procedure. A Baseline angiogram showing a calcified stenosis of the proximal and mid LAD. B Lesion preparation with rotational atherecomy using the 1.75 burr. C Shows final result after

the distal BRS and $1.98 \mathrm{~mm}^{2}$ at the proximal one (Fig. 2). A scoring balloon angioplasty using a $3.0 \times 10 \mathrm{~mm} \quad$ AngioSculpt $^{\mathrm{TM}}$ scoring balloon (AngioScore) was performed followed by $3.5 \times 20$ and $3.0 \times 20 \mathrm{~mm}$ Pantera $\mathrm{Lux}^{\mathrm{TM}}$ (Biotronik) paclitaxel eluting balloons (DEB) in the proximal and distal scaffolds, respectively. OCT clearly showed the neointimal cuts achieved by the scoring elements (white arrowheads, Fig. 3), neointimal disruption with some intraluminal material (yellow arrowheads, Fig. 3)

implantation of two $3 \times 28 \mathrm{~mm}$ bioresorbable scaffolds, with a good result in the final intravascular ultrasound (D)

and lumen expansion. At 2 years after balloon angioplasty (almost 3 years after BRS implantation), the patient remained asymptomatic and a scheduled angiographic control showed a patent vessel with no significant lesions. OCT showed favorable evolution of the neointimal damage caused by the scoring balloon and maintenance of the initial lumen expansion, with partial resorption of the scaffold struts for which footprints were still visible (Fig. 4). 

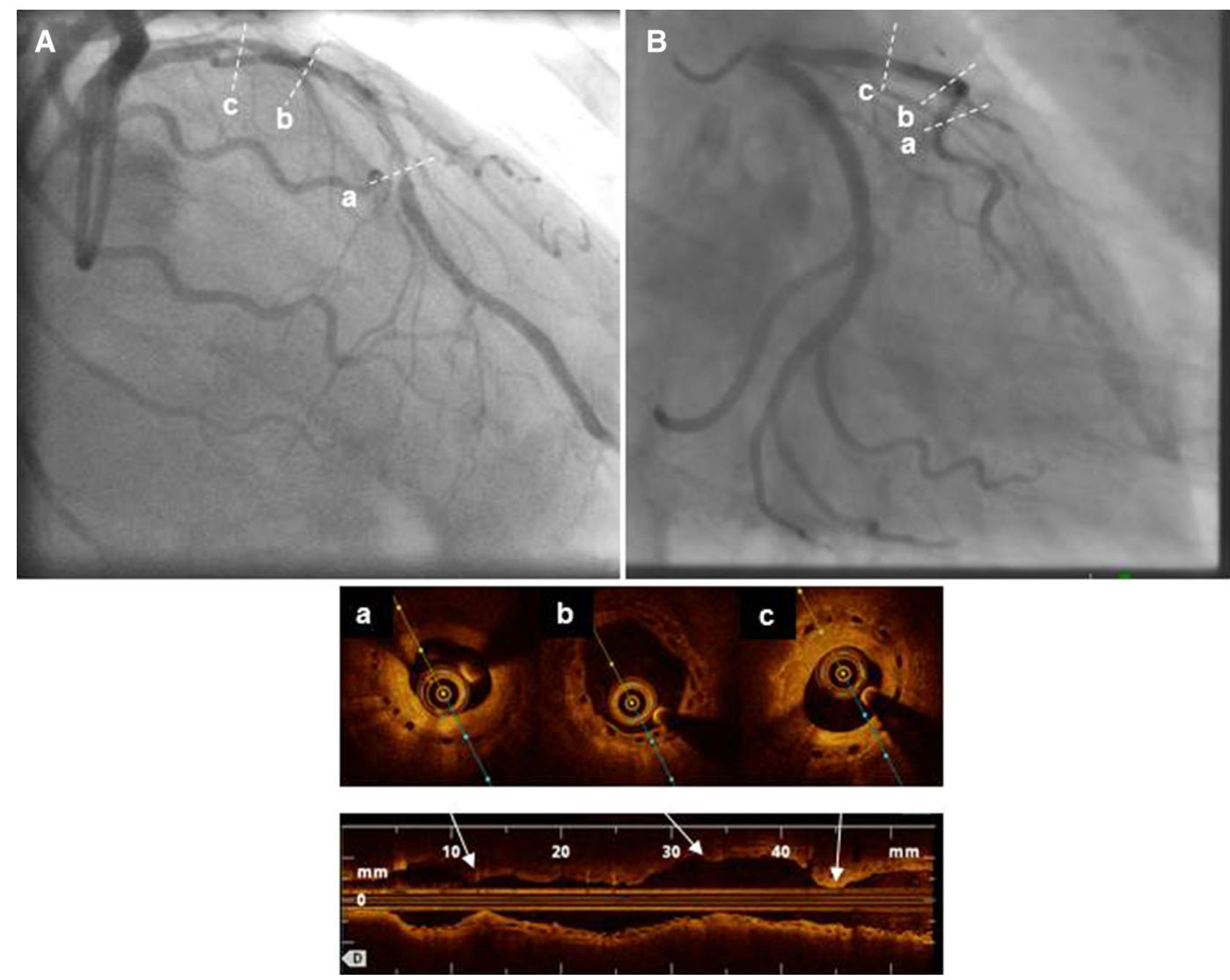

Fig. 2 In-scaffold restenosis. In A, B, the angiogram shows diffuse in-scaffold restenosis in the proximal and distal scaffolds. B is the final angiographic appearance after scoring balloon/drug-eluting balloon treatment. Optical coherence tomography (OCT) images $(a)$ and $(c)$ show homogeneous intra-scaffold tissue. The $b$ represents OCT cross section of the overlapping zone

\section{Compliance with Ethics Guidelines}

Informed consent was obtained from the patient for being included in the study.

\section{DISCUSSION}

The most important proposed advantage of BRS over the current metallic stent technology is to restore the natural physiologic vasomotor function and to hinder late thrombotic events. In our case, the initial treatment strategy was using RA in disrupting the continuity of intravascular calcium rings facilitating BRS implantation and potentially achieving a stent-free vessel in the long-term.

$\mathrm{RA}$ is a safe and effective method for lesion preparation before stent implantation [1] and its use could be extrapolated before BRS implantation, where lesion preparation is of utmost importance. However, additional vessel injury with RA has been previously suggested to be associated with increased late lumen loss [2]. In our case, this mechanism appears very likely because the in-scaffold restenosis was diffuse and affected both implanted BRSs.

The rate of target-lesion revascularization ranges from $1 \%$ to $3.3 \%$ after $\mathrm{BRS}$ 

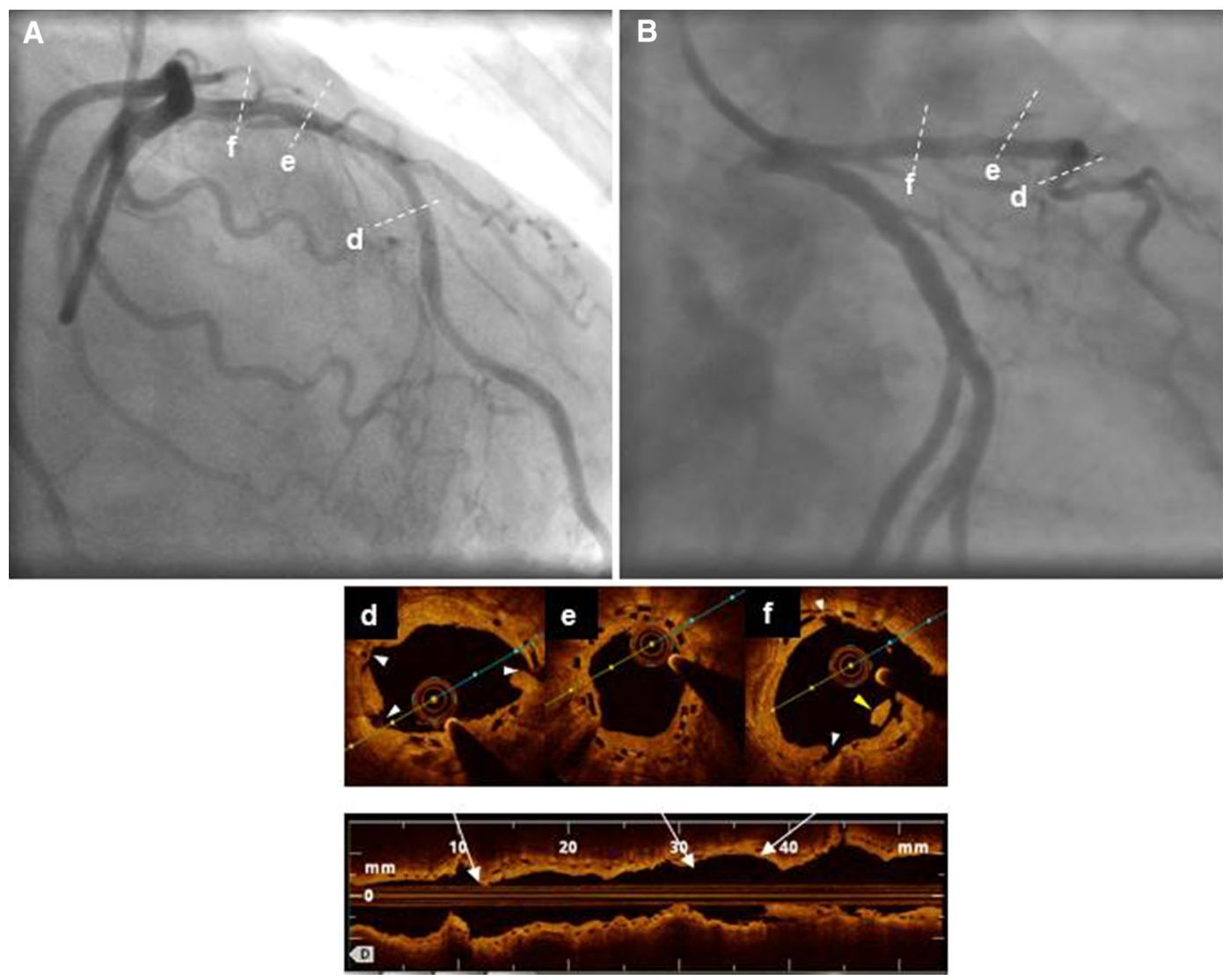

Fig. 3 Final result after scoring balloon/drug-eluting balloon treatment. In $\mathbf{A}, \mathbf{B}$, the final angiographic appearance after balloon-only treatment of the restenosis. $d-f$ Lumen expansion is clearly visible via optical coherence

implantation [3, 4]. The most often used method for the treatment of in-scaffold restenosis is a drug-eluting stent (DES) [5], but there is no sufficient data supporting this approach, and the optimal treatment strategy is still uncertain. Drug-eluting balloons have demonstrated their efficacy in treatment of restenosis following metallic stent implantation [6]. Additionally, scoring balloon predilatation prevents balloon slippage and enhances local plaque disruption, and its use in combination with DEB angioplasty improved angiographic results in the ISAR-DESIRE 4 Study [7]. In this reported case, by adopting the scoring/paclitaxel-coated balloon strategy, we tried avoiding a metallic tomography (OCT). The white arrowheads show the lumen-surface marks achieved by the scoring elements. Intraluminal material caused by neointimal dissection is also observed (yellow arrowhead)

stent implantation targeting to maintain the long-term benefits of BRS. The entire resorption process of BRS takes approximately 3 years to complete. However, between 3 and 6 months and as a consequence of hydrolysis of ester bonds, the device loses its structural continuity, and the radial strength decreases considerably until it is lost [8]. This loss of radial force is suspected to be source of recoil when balloon dilatation is performed alone after 6 months. In this patient, the vessel wall 10 months after BRS implantation opposed minimal recoil to the scoring/DEB treatment, which achieved sufficient stable lumen size and remained effective until 2 years of follow-up. 


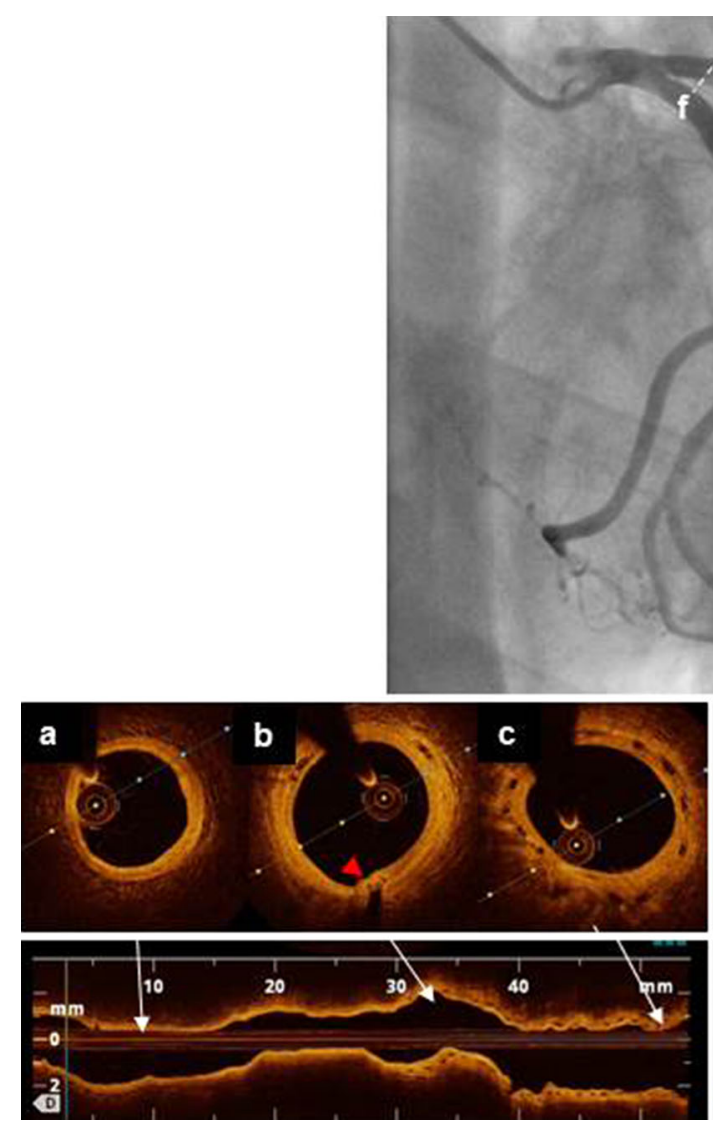

Fig. 4 Follow-up angiogram and optical coherence tomography (OCT) 2 years after treatment of the in-scaffold restenosis. $a$ is done in a zone of muscular bridging showing the absence of significant stenosis in that area. The red arrowhead shows the metallic marker of the distal implanted

In our case we tried to highlight the possibility of a balloon-only treatment of a restenosed bioresorbable scaffold. With an adequate initial lesion preparation, this strategy can be even applied in severely calcified coronary lesions. Finally, given the limited data, there is a need for further examination of the place of RA before BRS implantation and the optimal treatment strategy for in-scaffold restenosis from future RCTs.

\section{ACKNOWLEDGMENTS}

No funding or sponsorship was received for publication of this article. All named authors
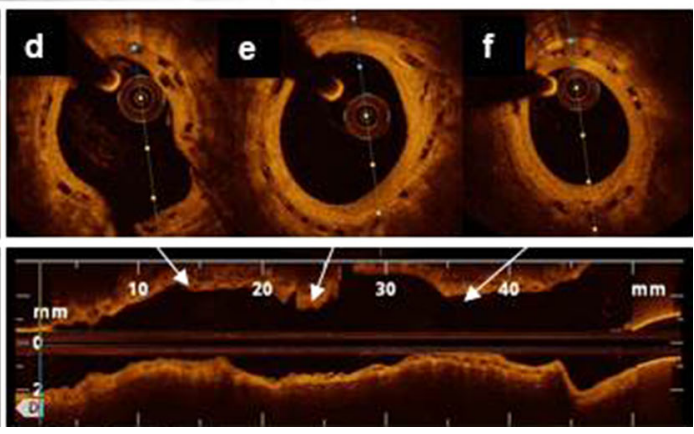

scaffold. The favorable evolution with disappearance of the neointimal damages caused by the scoring balloon is clear in the OCT images $b-f$, which also show partial resorption of the scaffold struts

meet the International Committee of Medical Journal Editors (ICMJE) criteria for authorship for this manuscript, take responsibility for the integrity of the work as a whole, and have given final approval to the version to be published.

Disclosures. G. Richardt reports receiving an institutional research Grant from Biotronik and St. Jude Medical. G. Richardt is also an advisory board member for Abbott Vascular and Boston Scientific and reports receiving lecture fees from Abbott Vascular, Biotronik, Boston Scientific, and Edwards Lifesciences. M. Abdel-Wahab is a proctor for Boston Scientific and reports receiving an institutional research grant from 
Biotronik and St. Jude Medical. A. Allali has no conflicts of interest to declare.

Compliance with Ethics Guidelines. Informed consent was obtained from the patient for being included in the study.

Open Access. This article is distributed under the terms of the Creative Commons Attribution-NonCommercial 4.0 International License (http://creativecommons.org/licenses/ by-nc/4.0/), which permits any noncommercial use, distribution, and reproduction in any medium, provided you give appropriate credit to the original author(s) and the source, provide a link to the Creative Commons license, and indicate if changes were made.

\section{REFERENCES}

1. Barbato E, Carrié D, Dardas P, et al. European expert consensus on rotational atherectomy. EuroIntervention. 2015;11:30-6.

2. Abdel-Wahab M, Richardt G, Joachim Büttner $H$, et al. High-speed rotational atherectomy before paclitaxel-eluting stent implantation in complex calcified coronary lesions: the randomized ROTAXUS (Rotational Atherectomy Prior to Taxus Stent Treatment for Complex Native Coronary Artery Disease) trial. JACC Cardiovasc Interv. 2013;6:10-9.

3. Ellis S, Kereiakes D, Metzger DC, et al. Everolimus-eluting bioresorbable scaffolds for coronary artery disease. N Engl J M. 2015;373:1905-15.

4. Lu C, Filion K, Eisenberg M. The safety and efficacy of absorb bioresorbable vascular scaffold: a systematic review. Clin Cardiol. 2016;39:48-55.

5. Nakatani S, Onuma Y, Ishibashi Y, et al. Early (before 6 months), late (6-12 months) and very late (after 12 months) angiographic scaffold restenosis in the ABSORB Cohort B trial. EuroIntervention. 2015;10:1288-98.

6. Byrne R, Neumann F-J, Mehilli J, et al. Paclitaxel-eluting balloons, paclitaxel-eluting stents, and balloon angioplasty in patients with restenosis after implantation of a drug-eluting stent (ISAR-DESIRE 3): a randomised, open-label trial. Lancet. 2013;381:461-7.

7. Byrne R-A, Kufner S, Joner M, et al. Neointimal modification with scoring-balloon and efficacy of drug-coated balloon therapy in patients with restenosis in drug-eluting coronary stents (ISAR-DESIRE 4 Study). San Francisco: Tct; 2015.

8. Oberhauser J, Hossainy S, Rapoza R. Design principles and performance of bioresorbable polymeric vascular scaffolds. EuroIntervention. 2009;5(Suppl F):F15-22. 